\title{
Validation of Italian multiple sclerosis quality of life 54 questionnaire
}

Alessandra Solari, Graziella Filippini, Laura Mendozzi, Angelo Ghezzi, Stefania Cifani, Elena Barbieri, Silvana Baldini, Andrea Salmaggi, Loredana La Mantia, Mariangela Farinotti, Domenico Caputo, Paola Mosconi

Istituto Nazionale Neurologico "C Besta", Milan, Italy A Solari

G Filippini

A Salmaggi

L La Mantia

M Farinotti

Fondazione Pro Juventute Don C Gnocchi, Milan, Italy

L Mendozzi

E Barbieri

D Caputo

Centro Studi Sclerosi Multipla, Gallarate,

VA, Italy

A Ghezzi

$S$ Baldini

Istituto di Ricerche Farmacologiche "M Negri", Milan, Italy

S Cifani

P Mosconi

Correspondence to:

Dr Alessandra Solari, Istituto

Nazionale Neurologico C

Besta, Laboratory of

Epidemiology, via Celoria 11,

20133 Milan, Italy.

Telephone 0039022394

391; fax 0039027063

8217; email:

neuroepidemiologia

(a) istituto-besta.it

Received 14 July 1998 and in final form

17 February 1999

Accepted 19 February 1999
Abstract

Objectives-Health related quality of life (HRQOL) inventories are multidimensional measures of patient-centred health status developed for clinical research. The MS quality of life 54 (MSQOL-54) is an MS-specific HRQOL inventory originally devised for English speaking patients. It consists of a core measure, the 36-item short form health survey (SF-36) previously adapted into Italian, and 18 additional items exploring domains relevant to patients with MS (MS-18 module). The authors translated and culturally adapted into Italian the MS-18 module of the MSQOL-54 questionnaire, and clinically validated the whole questionnaire.

Methods-The MS-18 module was translated following the methodology of the International Quality of Life Assessment (IQOLA) project. The MSQOL-54 was validated in 204 consecutive patients with MS seen between April and September 1997 at three participating centres. The questionnaire was explained by the physician who also administered the expanded disability status scale (EDSS) and mini mental status scale examination, and the patient filled in the MSQOL-54 and Beck depression inventory questionnaires (BDI), with assistance if required. The contribution of impairments and disabilities to MSQOL-54 scores were assessed, and mean scores were compared with normative data for the general Italian population, and with the original sample of United States MS patients.

Results-The mean age of the 204 patients was 42 years; mean EDSS score was 4.5 (range 0-8.5). Patients' participation in the assessment was satisfactory, and all scales satisfied the usual psychometric standards. The characteristics of the United States sample matched those of our patients in all but gender $(72 \%$ United States patients $v \mathbf{5 2 \%}$ Italian patients were women), and education (90\% United States patients and $44 \%$ Italian patients completed high school); MSQOL-54 profiles were also similar. The EDSS was significantly associated with the physical health composite but not with the mental health composite score. Multiple linear regression modelling showed that age and BDI independently predicted physical health composite $(p<0.001)$, and mental health composite $(p<0.001)$. Clinical worsening in the previous year had an independent effect on the physical health composite $(p<0.001)$.

Conclusions-The Italian version of MSQOL-54 is easy to administer and is well accepted by patients. Neurological impairment has a limited influence on perceived quality of life, while age and depressive symptoms has a major influence. (F Neurol Neurosurg Psychiatry 1999;67:158-162)

Keywords: multiple sclerosis; quality of life; outcome measures

Over the past decade health related quality of life (HRQOL) instruments have become increasingly popular as end point tools in clinical studies for measuring patient-assessed health status. They are also used for evaluating the impact of interventions, particularly in oncology and cardiology. ${ }^{12}$ When the goal of treatment is to improve patients' wellbeing rather than to increase survival, as in chronic conditions, HRQOL measurement is essential. More than 20 years of research have led to the consensus notion of quality of life as a multidimensional concept which usually investigates four healthrelated domains (physical, social, psychological/ emotional functions, and physical/emotional role). ${ }^{3}$ These areas are generally assessed by a generic instrument such as SF-36, while specific instruments assess the impact of the disease and the treatments given; almost all are aimed at English speaking patients. ${ }^{23}$

Research on quality of life assessment in MS is still limited. The few available studies have employed generic HRQOL inventories in MS patients, only recently have disease-specific instruments begun to be used. ${ }^{4-9}$ In Italy the usual approach to quality of life assessment is either to develop ex novo questionnaires, or to translate and culturally adapt those originally developed in English speaking countries. The first approach requires time, can be expensive, and is used when a reliable instrument suitable for translation and cultural adaptation is not available. Several generic instruments have been successfully adapted into Italian, such as the Nottingham health profile, and the Rotterdam symptoms check list. ${ }^{11}$

The International Quality of Life Assessment (IQOLA) project, which was launched in 1990, translated, adapted and validated the SF-36 questionnaire into 42 different languages. ${ }^{12}$ Recently the SF-36 was supplemented by 18 additional items (MS-18 module) to obtain the MS quality of life 54 (MSQOL-54) questionnaire specific for MS patients. ${ }^{4}$ We have now 
translated and culturally adapted the MS-18 module of the MSQOL-54 questionnaire, and validated the whole questionnaire on a group of 204 Italian MS patients. We also investigated clinical and sociodemographic characteristics in order to identify key factors affecting HRQOL.

\section{Methods}

INSTRUMENTS

The SF-36 questionnaire is one of the most widely used HRQOL instruments in the United States. It was devised to satisfy the minimum psychometric standards necessary for group comparisons involving general health dimensions (not specific to age, disease, or treatment group). ${ }^{12-16}$ SF-36 measures two major health concepts by means of two composite scores (physical health composite, and mental health composite) obtained from 8 multi-item scales: physical function; role limitation - physical; bodily pain; general health; vitality; social function; role limitation - emotional; and mental health; in all there are 36 items. An additional question is also present which asks about self-evaluated change in health status. SF-36 has been available in Italian since 1990 and data from a representative sample of the Italian population are available. ${ }^{17} 18$

The MS-18 module, originally devised in the United States in 1995 adds 18 additional items to SF-36 concerned with the following areas: health distress; sexual function; satisfaction with sexual function; overall quality of life; cognitive function; and energy. The composite instrument, composed of SF-36 and MS-18, is MSQOL-54 which contains 52 items grouped into 12 scales, plus two lone items. ${ }^{4}$

TRANSLATION AND ADAPTATION

A procedure similar to that used by the IQOLA project was adopted to translate and adapt the MS-18 module. ${ }^{12}{ }^{18}{ }^{19}$ Briefly, the steps involved were: (a) Translation and evaluation to ensure semantic equivalence and acceptability. A professional translator with experience with health and quality of life terminology but not with the MS-18, produced a translation. (b) During an initial meeting with quality of life assessment experts and clinicians, a version with a list of possible alternatives for the most controversial item stems and response choices was drawn up. Problematic items and response choices were retranslated into Italian from the original and a consensus version was arrived at. (c) Subsequently the questionnaire was filled in by three patients, the results of which were discussed at a second meeting that included patients and their proxies. (d) The final version of the questionnaire then emerged and was tested on 20 MS patients.

\section{PATIENTS}

Between April and September 1997, all MS patients presenting at the three Institutes involved in the study were considered for inclusion. Eligible admitted patients and outpatients satisfied the following criteria: clinically definite or laboratory supported MS, age 18 years or over, written informed consent, and knowledge of their MS diagnosis. Exclusion criteria were: exacerbation in the month prior to enrolment, and one or more overt additional illnesses. ${ }^{20}$

\section{ADMINISTRATION OF THE QUESTIONNAIRE}

The MSQOL-54 questionnaire was explained to the patient by the physician, who also administered the expanded disability status scale (EDSS) and mini mental state examination (MMSE). ${ }^{21-23}$ The patient then filled in the questionnaire, with help if there were difficulties in reading or writing. The patient was then asked two further questions regarding the relevance and clarity of the questions; the patient's views and suggestions were also sought and noted. The time required by each patient to complete the questionnaire was noted. The short form of the Beck depression inventory (BDI) was then given to the patient to compile. This consists of 13 items that explore feelings and attitudes relating to general depressive status. ${ }^{24}$ The examining physicians received prior training in order to standardise administration of the scales.

STATISTICAL ANALYSIS

MSQOL-54 scale scores were assembled using the Likert method for summed ratings and the raw scores were linearly transformed into 0-100 scales: the higher the transformed score, the better the patient's HRQOL. ${ }^{14}$ Patient acceptability was assessed from mean time required to complete the questionnaire; the proportion of missing data at item and scale level; and whether assistance was required to complete the questionnaire. In addition, the response consistency index was applied to calculate the percentage of logical inconsistencies committed in completing the questionnaire. This analysed responses using internal checks based on answers to 15 pairs of items. For example, being able "to walk a kilometre" but "not a hundred meters" is considered an inconsistency in scoring the response consistency index. ${ }^{14} 1518$

Grouping and scaling assumptions were assessed using standard psychometric instruments described in the papers that presented the original questionnaire. ${ }^{14-16}$ Briefly, as the questionnaire is based on a multidimensional conceptualisation of health, the multitrait analysis approach was adopted to test whether conceptualisation into domains fitted the data, and whether the results of the Italian questionnaire replicated the results obtained with the English language questionnaire in terms of convergence, divergence and internal consistency reliability (Cronbach's $\alpha$ ). ${ }^{25-27}$

Clinical validity was assessed comparing mean MSQOL-54 scores by patient's age, severity of MS symptoms over the preceding year, EDSS score, BDI score, MMSE score, disease duration, education, marital status, and current employment status. The Wilcoxon rank-sum test and Kruskal-Wallis test were employed for these comparisons. Univariate and multivariate linear regression were used to investigate the relationship between the composite scores and clinical covariates. Of the continuous explanatory variables, disease duration, 


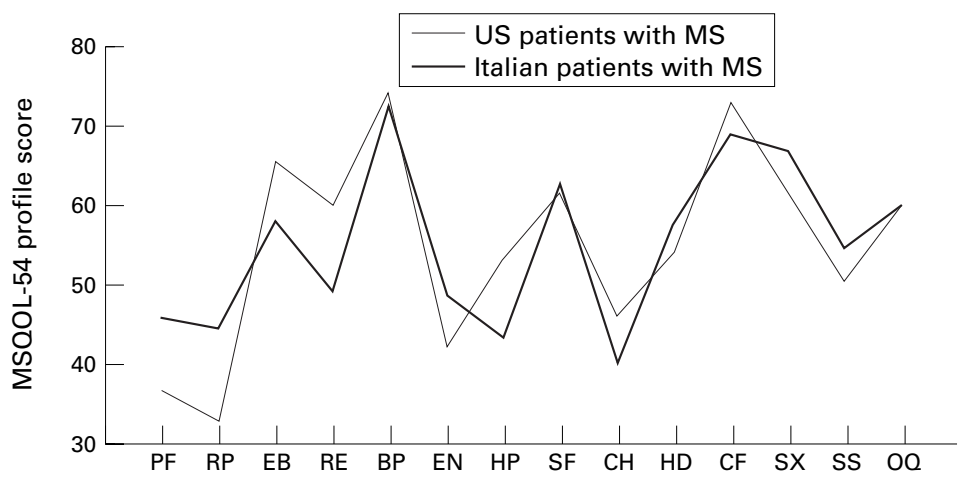

Comparison of MSQOL-54 profiles between 179 US MS patients and 204 Italian MS patients (bold line). $P F=$ physical function; $R P=$ role limitation - physical; $E B=$ emotional well being; $R E=$ role limitation - emotional $E N=$ energy; $H P=$ health perception; $S F=$ social function; $C H=$ change in health; $H D=$ health distress; $C F=$ cognitive function; $S X=$ sexual function; $S S=$ satisfaction with sexual function; $O Q=$ overall quality of life.

EDSS, MMSE, and BDI scores did not follow a normal distribution, and were transformed into categories; age was normally distributed, and considered a continuous variable. The other covariates included in the regression analysis were categorical. Finally, we compared qualitatively the Italian health profiles with those from the original United States survey (fig).

\section{Results}

Of 405 patients seen at the three participating centres between April and September 1997, 204 were enrolled. Of the remaining 201 patients, $46(23 \%)$ had concomitant diseases, $64(32 \%)$ were in exacerbation, $76(38 \%)$ had no knowledge of their diagnosis or had a disease duration of less than 6 months, and 15 $(7 \%)$ did not want to participate. Ninety four

Characteristics of the 204 patients included in the study

\begin{tabular}{|c|c|}
\hline Characteristic & Number (\%) \\
\hline Men & $98(48)$ \\
\hline Age $(y) \rrbracket$ & $\begin{array}{l}42.6 \pm 11.2 \\
(19-67)\end{array}$ \\
\hline Hospitalised patients & $94(46)$ \\
\hline \multicolumn{2}{|l|}{ Education (y): } \\
\hline Elementary $(1-8)$ & $87(42)$ \\
\hline High school (9-13) & $91(45)$ \\
\hline College, master $(14+)$ & $26(13)$ \\
\hline \multicolumn{2}{|l|}{ Current employment status: } \\
\hline Employed outside the home/student & $72(35)$ \\
\hline Homebound employment & $15(7)$ \\
\hline Homemaker & $31(15)$ \\
\hline Sheltered work & $9(4)$ \\
\hline Retired & $59(29)$ \\
\hline Unemployed & $18(9)$ \\
\hline \multicolumn{2}{|l|}{ Marital status: } \\
\hline Single (never married) & $53(26)$ \\
\hline Married/cohabiting & $134(66)$ \\
\hline Widowed & $5(2)$ \\
\hline Separated/divorced & $11(5)$ \\
\hline Disease duration $(y) \subseteq$ & $12.8 \pm 8.9(1-41)$ \\
\hline \multicolumn{2}{|l|}{ Clinical worsening in the last year: } \\
\hline Absent $/$ mild & $145(73)$ \\
\hline Moderate/severe & $54(27)$ \\
\hline \multicolumn{2}{|l|}{ Current disease course } \\
\hline Relapsing remitting & $79(40)$ \\
\hline Relapsing progressive & $69(35)$ \\
\hline Chronic progressive & $48(25)$ \\
\hline Financial compensation requested & $102(50)$ \\
\hline EDSS score & $4.5 \pm 2.1(0-8.5)$ \\
\hline MMSE score & $\begin{array}{l}26.9 \pm 2.5 \\
(14.6-30.3)\end{array}$ \\
\hline BDI score & $8.1 \pm 6.5(0-33)$ \\
\hline
\end{tabular}

$\S$ Mean \pm SD (min-max)

EDSS $=$ the expanded disability status scale $;$ MMSE $=$ the min mental status scale examination; BDI $=$ the Beck depression index, short form patients (46\%) were hospitalised (table). Mean age was 43 years (range 19-67), 98 (48\%) were men; $134(66 \%)$ were married and $91(45 \%)$ had completed high school (up to 13 years of schooling). Mean age at disease onset was 30 years (range 11-51), average duration of MS was 13 years (range $0.5-41$ ), and mean EDSS score was 4.5 (range 0-8.5). Mean time to complete the questionnaire was 19 minutes (range 5-60 minutes). Seventy eight patients $(38 \%)$ needed help; 17 of whom (22\%) required help reading some or all the items: nine $(12 \%)$ required help marking the form; 22 $(28 \%)$ required both reading and writing assistance; and $30(38 \%)$ required explanation of one or more items.

The percentage of missing data was low $(0.5 \%-2.9 \%$ at the item level) except for items concerning sexual function and satisfaction with sexual function, where high percentages of data were missing (both 19\%). An analysis to identify a pattern in the missing responses was performed; no pattern emerged, however higher percentages of missing responses occurred in items assessing sex (sexual function and satisfaction with sexual function scales), independently of the clinical and demographic characteristics of the patients.

The results of consistency testing were less satisfactory: there were no logical inconsistencies in only $66 \%$ of completed forms, however in $82 \%$ of all forms there was one or less inconsistency. Finally, over $90 \%$ of the patients did not consider that the questionnaire was difficult to understand or that it contained embarrassing questions.

The MSQOL-54 scales satisfied the usual psychometric standards. ${ }^{13}{ }^{14}$ Within scale coefficients were homogeneous and higher than 0.40 in most instances (indicating good convergent validity); greater item scale correlations were found within scale than between scales (indicating good discriminant validity). Internal consistency reliability indexes were greater than 0.70 for all scales except sexual function. The detailed psychometric results are available in annotated table form on request from the authors.

Clinical validity was assessed comparing mean MSQOL-54 physical health composite and mental health composite scores by patient's age, gender, severity of MS symptoms over the preceding year, EDSS score, BDI score, MMSE score, disease duration, education, marital status, and current employment status. Marital status was associated with the physical and mental health composite scores, with married or cohabiting patients scoring worse (mean (SD) physical health composite, 50.5 (18); mean mental health composite, 53.9 (22) than single, widowed, divorced or separated patients (mean physical health composite, 62.2 (18); mean mental health composite, 65.1 (21) (p < $0.001)$.). Other factors negatively affecting both composite scores were depressed mood $(\mathrm{p}<$ 0.001 ), disease duration over 10 years ( $\mathrm{p}<$ 0.005 ), clinical worsening in the previous year (physical health composite, $\mathrm{p}<0.001$; mental health composite, $p=0.003$ ), and age $>50$ years (physical health composite, $\mathrm{p}<0.001$; mental 
health composite, $\mathrm{p}=0.01)$. Patients scoring higher on EDSS had lower physical health composite score (mean physical health composite: 62.4 (18) for patients with EDSS $\leqslant 3.5 ; 50.7$

(17) for patients with EDSS 4.0-6.0, and 43.4

(16) for those with EDSS $>6.0 ; p<0.001$ ). Full employment status has a positive impact on the physical health composite $(p=0.03)$. Gender, cognitive status as assessed by the MMSE and education did not influence any composite score.

Univariate and multivariate linear regression were used to investigate the relationship between the composite scores and clinical covariates. By univariate analysis there was a highly significant inverse relationship between patient age and physical health composite score. EDSS and BDI scores were also inversely related to physical health composite. Marital status was significantly predictive of physical health composite, with single, widowed, divorced or separated patients having higher scores than married or cohabiting patients $(\beta=11.7,95 \% \mathrm{CI}, 5.7$ to 17.7). A clinical worsening in the previous year was associated with lower physical health composite score ( $\beta=-14.4,95 \% \mathrm{CI},-19.8$ to -8.9) and disease duration had a weak negative association with this score. Patients who completed high school or university, were fully employed, and had higher MMSE scores had higher physical health composite scores, but the differences were not statistically significant. The final multivariate regression model obtained explained almost $50 \%$ of the variance, and included BDI, age, and clinical worsening as independent explanatory variables. EDSS score, marital status and disease duration did not account for significant proportions of the variance, and were thus removed from the model.

By univariate analysis, BDI score was inversely predictive of mental health composite score. Other explanatory variables inversely and significantly associated with mental health composite score were age, clinical worsening in the previous year, disease duration, and marital status. The multivariate model retained BDI and age as independent explanatory variables accounting for almost $40 \%$ of the variance. The detailed results of the regression are available on request.

We compared mean scale scores for the whole sample with the United States MS patients' sample (figure). The characteristics of the United States sample were similar to those of our patients in terms of age (mean age of United States patients 45 years, range $20-67$; v mean age of Italian patients 43 years, range 19-67 years); marital status (59\% v 56\% married); working status (39\% v 35\% employed outside home); and disease duration (mean duration $13.2 v 12.8$ years; range $1-40 v 1-41$ years). However, these two populations differed in terms of gender $(72 \%$ United States patients $v 52 \%$ Italian patients were women), and education (98\% $v 45 \%$ completed high school). Considering the MSQOL-54 profile (figure) there was a mean difference of more than 10 points between United States and Italian patients for role limitation- physical (32.9 v
44.6) and role limitation-emotional (60.0 v 49.3). Scores for the remaining scales were similar in the two groups of patients.

\section{Discussion}

The Italian MSQOL-54 questionnaire was easy to administer and well accepted by our MS patients, with $90 \%$ of them stating that no embarrassing or difficult to interpret questions were present. The results were similar to those obtained using the original questionnaire on United States MS patients. Nevertheless it is important to underline that it took an average of 20 minutes to complete the questionnaire, with more disabled patients taking longer. Help was necessary in $40 \%$ of all cases, a proportion that increased in relation to poorer clinical status. The response consistency index revealed a greater number than expected of logical inconsistencies compared to the patients with other chronic diseases (no logical inconsistencies in $83 \%$ of completed forms in patients with endstage renal disease and in $78 \%$ in patients with chronic obstructive pulmonary disease).${ }^{18}{ }^{28} \mathrm{On}$ the basis of this finding, the use of proxy responders should be considered for the most clinically compromised MS patients.

The high percentage of missing answers to the items on sexual function and satisfaction with sexual function is an intriguing feature. This was also reported (to a lesser extent) in the United States survey, with 160 of 179 (89\%) patients completing enough items on the sexual function scale to enable scoring, and 150 of 179 (84\%) answering the single item question on satisfaction with sexual function. Our finding is consistent with data from other Italian surveys, and is probably attributable to Italian social and cultural characteristics. Previous studies that investigated HRQOL in Italian subjects decided not to explore these aspects. ${ }^{28-30}$

Age influenced both composite scores, retaining an independent effect in the multiple regression analyses in which physical health composite $\left(R^{2}=0.20\right)$ and mental health composite $\left(R^{2}=0.08\right)$ were outcome variables.

Depressive symptoms had a major influence on HRQOL. Patients with higher BDI scores had lower scores in all MSQOL-54 scales; and BDI score was a statistically significant predictor low scores in all scales (data not shown) and composite scales. In both the univariate and multivariate analyses, BDI score had a significant and independent effect on both composite scores (mental health composite, $R^{2}=0.34$; physical health composite, $R^{2}=0.29$ ). We used a scoring system to assess depressive symptoms not a structured interview, as psychiatric assessment was not the primary aim of the study. Depression can result from individual reactions to MS-associated disability and symptoms, as well as from the disease process itself. ${ }^{32}$ The reported lifetime occurrence of major depression in MS patients is between $42 \%$ and $54 \%^{32-34}$; the condition is often overlooked in MS patients and can be treated successfully. ${ }^{34}$

As expected, the effect of neurological impairment was captured by the physical health composite score. EDSS was also an independent variable inversely related to 
physical health composite in the regression analysis, however it was not statistically significant after adjusting for age, clinical worsening, and BDI.

Cognitive function, as assessed by MMSE, did not affect the composite scores. The regression analysis showed that MMSE score had only a small impact on mental health composite and this disappeared after adjusting for age and BDI in the multivariate model.

Disease duration over 10 years and clinical worsening in the previous year were significantly related to lower composite scores in univariate analyses, and in the multivariate model, clinical worsening retained its predictivity for lower physical health composite score.

An unexpected finding was that single, widowed, divorced, or separated patients had significantly higher health composite scores than married or cohabiting patients. In a larger study married and cohabiting patients could profitably be analysed separately. Marital status could be a confounder of other covariates (for example, patients might stay married or cohabit simply because they cannot live alone due to their disabilities); however in the multivariate model marital status did not persist as an independent predictor of physical and mental health composite scores after controlling for other explanatory variables, such as patient age.

In conclusion, our study has shown that the Italian version of MSQOL-54 is easy to administer and is well accepted by patients. Neurological impairment, as assessed by EDSS has a limited influence on perceived quality of life as measured by MSQOL-54, while age and depressive symptoms has a major influence. In a recently published study, MS patients and physicians disagreed on which domains of health status were most important for patients. ${ }^{35}$ We think that clinical trials and outcome studies should assess the effect of interventions taking into account, by means of accurate and reliable instruments, all components of health status that are considered important by the patients, and not simply rely on physician-assessed measures of disease status. As a part of a continuing research programme on HRQOL assessment in MS, we have devised an Italian proxy version of MSQOL-54. This should be useful for assessing the status of cognitively impaired patients, and also for overcoming problems of missing data and loss to follow up in clinical trials and longitudinal studies using MSQOL-54 as an end point. The findings of a study in which the proxy version was applied to surrogate informants (for example, health care providers and "significant others") to obtain complementary or additional information on patient HRQOL, will be published shortly.

This work was supported in part by Italian National Health Ministry (grant No $96 / \mathrm{J} / \mathrm{T} 55$, to AS). We are indebted to Drs Barbara Vickrey and Iris Gutmanis for allowing us to adapt the original version of MSQOL-54, to Dr Giovanni Apolone for his critical review of the manuscript, and to Don Ward for help with the English.

1 Spilker B, ed. Quality of life and pharmacoeconomics in clinical trials, 2nd ed. Philadelphia: Lippincott-Raven, 1994.

2 Patrick D, Deyo R. Generic and disease-specific measures in assessing health status and quality of life. Med Care 1989;27(suppl):217-32.
3 Hunt SM, McEwen J, McKenna SP, eds. Measuring health status. London: Croom Helm, 1986.

4 Vickrey BG, Hays RD, Harooni R, et al. A health-related quality of life measure for multiple sclerosis. Qual Life Res 1995;4:187-206.

5 Brunet DG, Hopman WM, Singer MA, et al. Measurement of health-related quality of life in multiple sclerosis patients. Can f Neurol Sci 1996;23:99-103.

6 Lankhost GJ, Jelles F, Smits RC, et al. Quality of life in multiple sclerosis: the disability and impact profile. 7 Neurol 1996;243:469-74.

7 Cella DF, Dineen MA, Arnason B, et al. Validation of the functional assessment of multiple sclerosis quality of life instrument. Neurology 1996;47:129-39.

8 Aaronson KJ. Quality of life among persons with multiple clerosis and their caregivers. Neurology 1997;48:74-80.

9 Pfennings LEMA, Cohen L, Van der Ploeg HM. Assessing the quality of life in patients with multiple sclerosis. In: Thompson AJ, Polman C, Hohlfeld R, eds. Multiple sclerosis: clinical challenges and controversies. London: Martin Dunitz 1997.

10 Hunt SM, McKenna SP, McEwen J, et al. The Nottingham health profile: subjective health status and medical consultations. Soc Sci Med 1981;15A:221-9.

11 de Haes JCJM, van Knippenberg FCE, Nejit JP. Measuring psychological and psychical distress in cancer patients: structure and application of the Rotterdam symptoms check-list. Br f Cancer 1990;62:1034-8.

12 Aaronson NK, Acquadro C, Alonso J, et al. International quality of life assessment (IQOLA) project. Qual Life Res 1992;1:349-51

13 Ware JE, Sherbourne CD. The MOS 36-item short form health survey (SF-36) I. Conceptual framework and item selection. Med Care 1992;30:473-83.

$14 \mathrm{McHorney}$ CA, Ware JE, Raczek AE. The MOS 36-item short form health survey (SF-36) II. Psychometric and clinical tests of validity in measuring physical and mental health constructs. Med Care 1993;31:247-63.

15 McHorney CA, Ware JE, Lu JFR, et al. The MOS 36-item short form health survey (SF-36) III. Tests of data quality, scaling assumptions, and reliability across diverse patients groups. Med Care 1994;32:40-66.

16 Ware JE, ed. SF-36 Health survey. Manual and interpretation guide. Boston: The Health Institute, New England Medical Center.

17 Apolone G, Cifani S, Liberati MC, et al. Questionario sullo stato di salute SF-36. Traduzione e validazione della versione italiana: risultati del progetto IQOLA. Medic 1997;5:86-94.

18 Apolone G, Mosconi P. The Italian SF-36. Health survey: translation, validation and norming from a clinical epidemiology perspective. 7 Clin Epidemiol 1998;51:1025-36.

19 Ware JE, Gandek BL, Keeler SD, the IQOLA project. Evaluating instruments used cross-nationally: methods from the IQOLA project. In: Spilker B, ed. Quality of life from the IQOLA project. In: Spilker B, ed. Quality of hife and pharmacoeconomics

20 Poser CM, Paty DW, Scheinberg L, et al. New diagnostic criteria for multiple sclerosis. Ann Neurol 1983;13:227-31.

21 Kurtzke JF. Rating neurologic impairment in multiple sclerosis: an expanded disability status scale. Neurology 1983;33:1444-52.

22 Folstein MF, Folstein SE, McHugh PR. "Mini-mental state": a practical method for grading the cognitive state of patients for the clinician. $\mathcal{F}$ Psychiatr Res 1975;12:189-98.

23 Measso G, Cavarzeran F, Zappalà G, et al. The mini-mental state examination: normative study of an Italian random sample. Developmental Neuropsychology 1993;9:77-85.

24 Beck AT, Beck RW. Screening depressed patients in family practice: a rapid technique. Postgrad Med 1972;52:81-5.

25 Hays RD, Hayashi T. Beyond internal consistency reliability: rationale and users guide for mutitrait analysis program on the microcomputer. Behavior Research Methods Instruments and Computers 1990;22:167-75.

26 Cronbach LJ. Coefficient $\alpha$ and the internal structure of tests. Psicometrika 1951;16:297-334.

27 Bland JM, Altman DG. Chronbach's $\alpha$. BMF 1997;317:572.

8 Apolone G, Mosconi P, Ware JE, eds. Questionario sullo stato di salute SF-36: manuale d'uso e guida all'interpretazione dei risultati. Milano: Guerini A ed associati, 1997.

29 The GISSI-Nursing. Valutazione della percezione della qualità di vita da parte del paziente con infarto miocardico. $G$ Ital Cardiol 1997;27:865-76.

30 Mingardi G, for the DIA-QOL Group. From the development to the clinical application of a questionnaire on the quality-of-life in dialysis: the experience of the Italian collaborative DIA-QOL (dialysis-quality of life) group. Nephrol Dial Transplant 1998;13:S70-5.

31 The GIVIO Investigators. Impact of follow-up testing on survival and health related quality of life in breast cancer
patients. A multicenter randomized controlled trial. $\mathscr{f} A M A$ 1994;271:1587-92.

32 Sadovnik AD, Remick RA, Allen J, et al. Depression and multiple sclerosis. Neurology 1996;46:628-32.

33 Joffe RT, Lippert GP, Gray TA. Depressions and multiple sclerosis. Arch Neurol 1987;44:376-8.

34 Minden S, Orav J, Reich P. Depression in multiple sclerosis. Gen Hosp Psichiatry 1987;9:426-34.

35 Rothwell PM, McDowell Z, Wong CK, et al. Doctors and patients don't agree: cross sectional study of patients and doctors perceptions and assessments of disability in multiple sclerosis. BMF 1997;314:1580-3. 